\title{
MARTINI Coarse-Grained Model of Triton TX-100 in Pure DPPC Monolayer and Bilayer Interfaces.
}

Antonio Pizzirusso $^{a}$, Antonio De Nicola $^{*}{ }^{a}$, Giuseppe Milano $^{a}$

${ }^{a}$ Dipartimento di Chimica e Biologia, Università di Salerno, I-84084 via Giovanni Paolo II, Fisciano (SA), Italy.

\section{Supporting Information}


In order to set-up the CG simulations to calculate the isotherm curves of pure DPPC monolayer at water/air interface, we employed the same set-up described by Duncan ${ }^{1}$. In particular, each CG system has been prepared by packing the lipids in a way that the initial area/DPPC was $0.64 \mathrm{~nm}^{2}$.Then, depending from the temperature at which the system has been simulated, a number of external surface pressure $\pi$ has been kept constant for the entire simulation time (Table S1).

Table S1. Values of surface pressure $\pi$ and surface tension $\gamma$ (see eq. 1) used in the N $\pi$ T simulations of pure DPPC monolayer. A time of $60 \mathrm{~ns}$ has been simulated for all systems listed in table.

\begin{tabular}{cccc}
$\begin{array}{c}\gamma \\
(\mathrm{mN} / \mathrm{m})\end{array}$ & $\begin{array}{c}\pi_{295.15 \mathrm{~K}} \\
(\mathrm{mN} / \mathrm{m})\end{array}$ & $\begin{array}{c}\pi_{298.15 \mathrm{~K}} \\
(\mathrm{mN} / \mathrm{m})\end{array}$ & $\begin{array}{c}\pi_{323.15 \mathrm{~K}} \\
(\mathrm{mNN} / \mathrm{m})\end{array}$ \\
\hline 1 & 71.5 & 71 & 66.9 \\
10 & 62.5 & 62 & 57.9 \\
25 & 47.5 & 47 & 42.9 \\
37.9 & - & - & 30.0 \\
40 & 32.5 & 32 & 27.9 \\
42.5 & - & 30.0 & - \\
42.8 & 30.0 & - & - \\
65 & 7.5 & 7 & 2.9 \\
70 & 2.5 & 2 & -2.5 \\
72.5 & 0.0 & -0.5 & - \\
73 & -0.5 & -1 & - \\
73.5 & -1 & -1.5 & - \\
74.0 & -1.5 & -2 & - \\
74.5 & -2 & -2.5 & - \\
75 & -2.5 & -3 & -7.5 \\
\hline
\end{tabular}

We reported in Figure S1 the comparison between the calculated (in the $N \pi T$ ensemble) and experimental isotherms for the pure DPPC monolayer at water/air interface. In Figure S1-A all isotherm curves calculated by employing the MARTINI force-field are reported. In order to test the 
effect of different ensembles (NPT and $N \pi T)$ on the behaviour of isotherm, an additional simulation at $298 \mathrm{~K}$ has been performed.
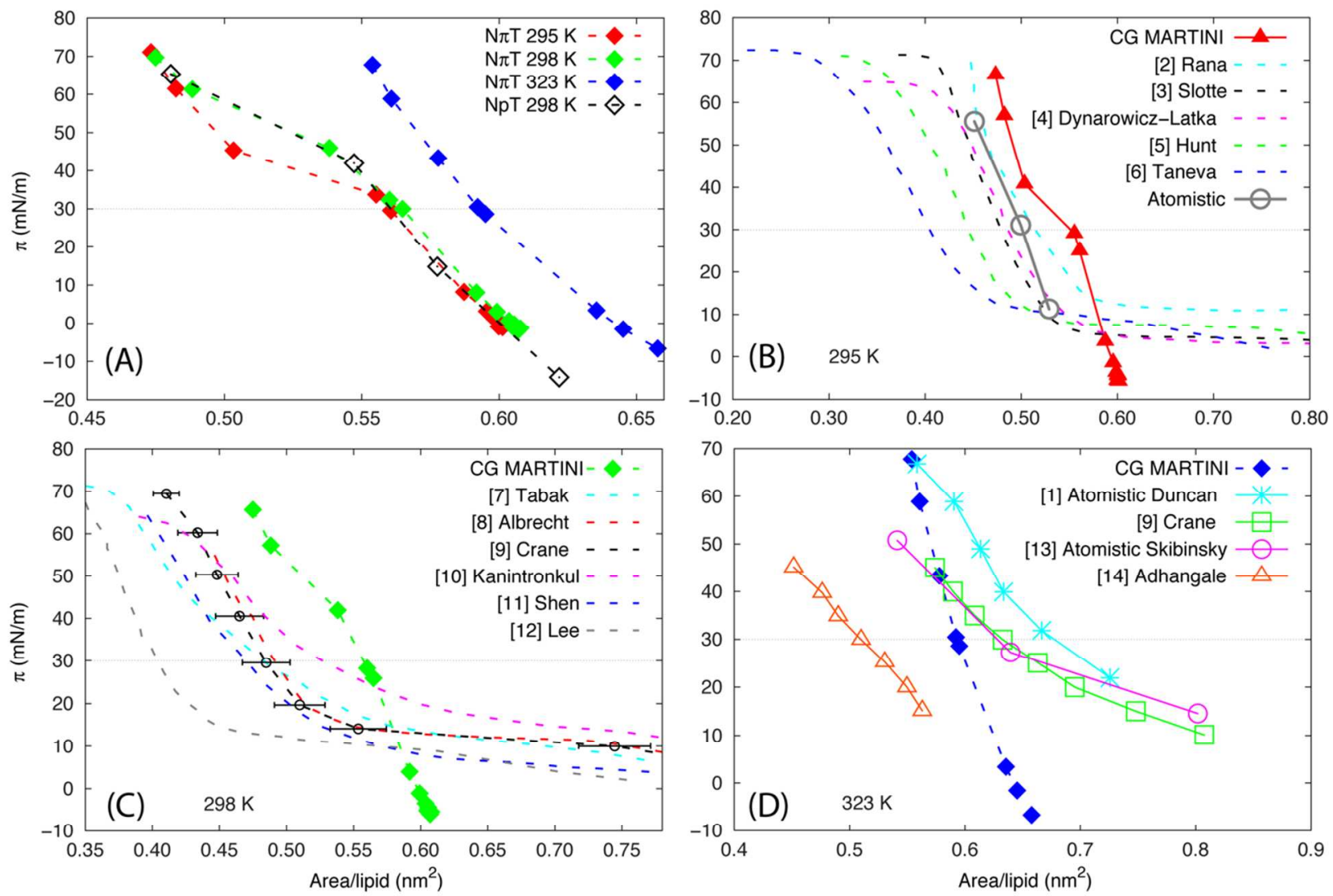

Figure S1. (A) Isotherms calculated at $2955^{2-6} 298^{7-12}$ and $325 \mathrm{~K}^{1,9,13-14}$ for pure DPPC monolayer system at water/air interface. Comparison between the calculated isotherm and experimental and simulated data are reported for (B) $295 \mathrm{~K}$ (C) $298 \mathrm{~K}$ (D) $323 \mathrm{~K}$. For each point an average over the last $20 \mathrm{~ns}$ of simulation time has been performed.

Due to the intrinsic diversities of experimental methods, and to different experimental conditions in the measurements of isotherms, as example different expansion/compression rate or different spreading solvent used in the measurements, the data comparison is not trivial. We reported the comparison of the calculated isotherm from MARTINI CG model with the respect to a range experimental measurements. The Percentage increment of area due to the insertion of Triton TX-100 in the DPPC monolayer is reported, in the following Figure S2, as function of both external surface pressure and TX-100 content. 

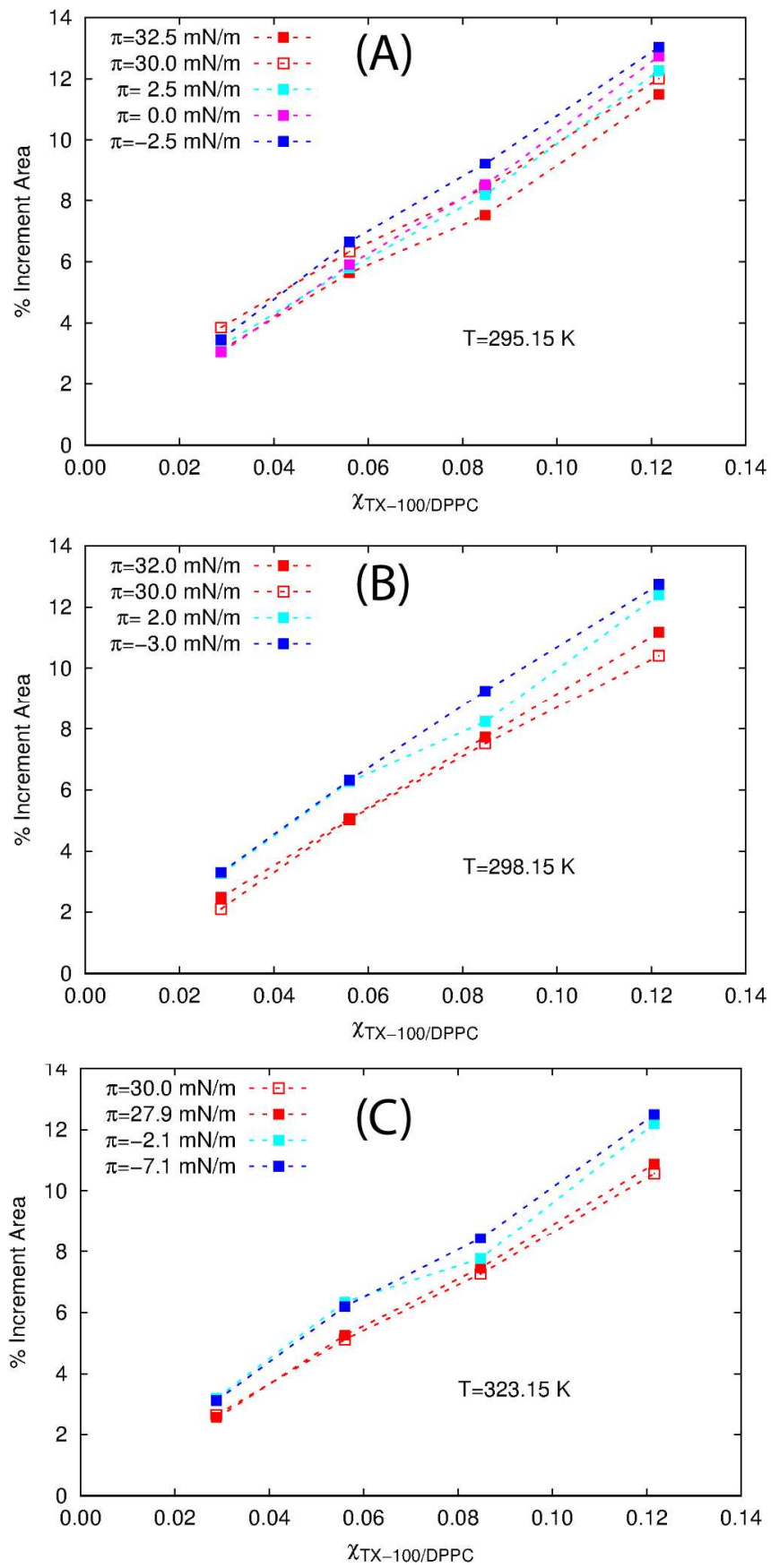

Figure S2: Percentage Increment of Area at: (A) 295.15 K, (B) 298 K, (C) 323 K. The description of the CG systems are reported in the Table 2 of the main text. 
Additional comparison of the results obtained from the CG simulation of bilayer system at $323 \mathrm{~K}$ are reported in the Figure S3. In particular, we compared the electron density profiles of the most relevant groups (Carbon tails, Carbons at the end of tails of DPPC molecule, and the PO4 group) with the respect to the profile reported by Marrink. ${ }^{15}$

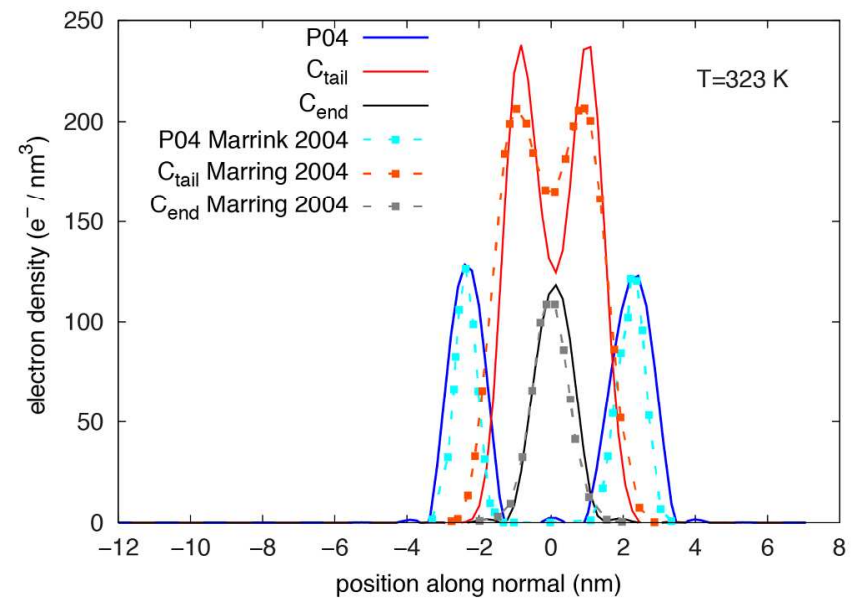

Figure S3: Comparison of the Electron Density Profile of DPPC bilayer simulated at $323 \mathrm{~K}$ employing the MARTINI model with the respect of the profile published by Marrink. ${ }^{15}$
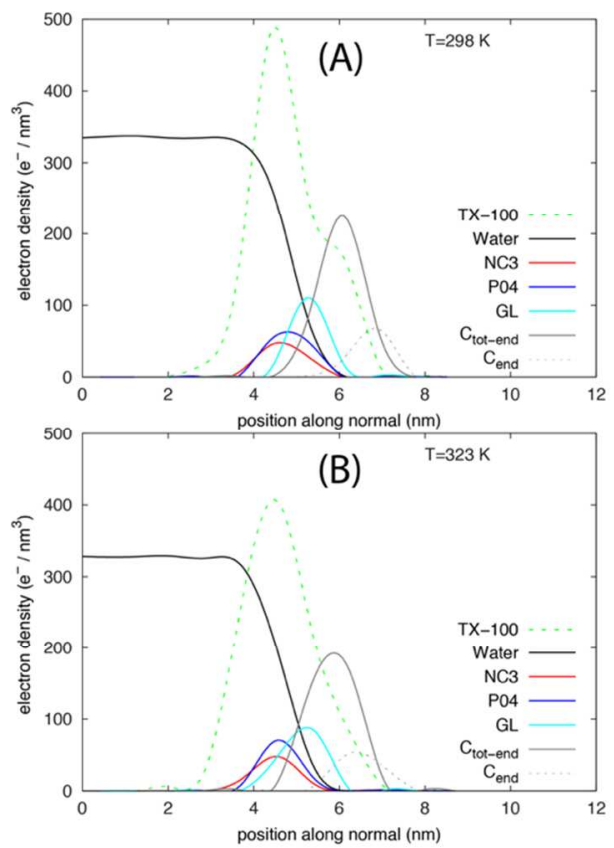

Figure S4: Electron Density Profiles calculated on CG monolayer systems (TX-100/DPPC $=0.122)$ at: (A) 298 and $(B)$ $323 \mathrm{~K}$ are reported. 


$$
\chi=0.056
$$
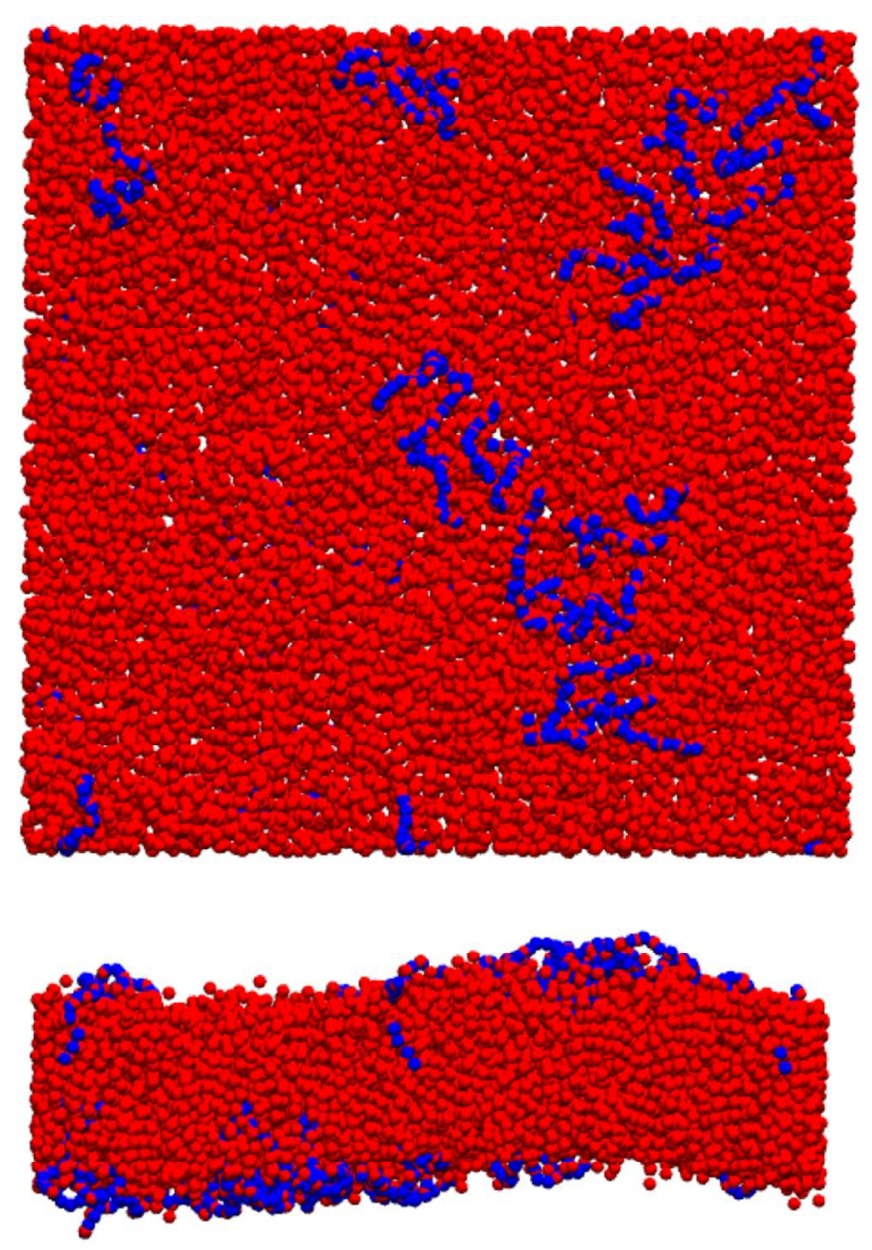

$\chi=0.085$
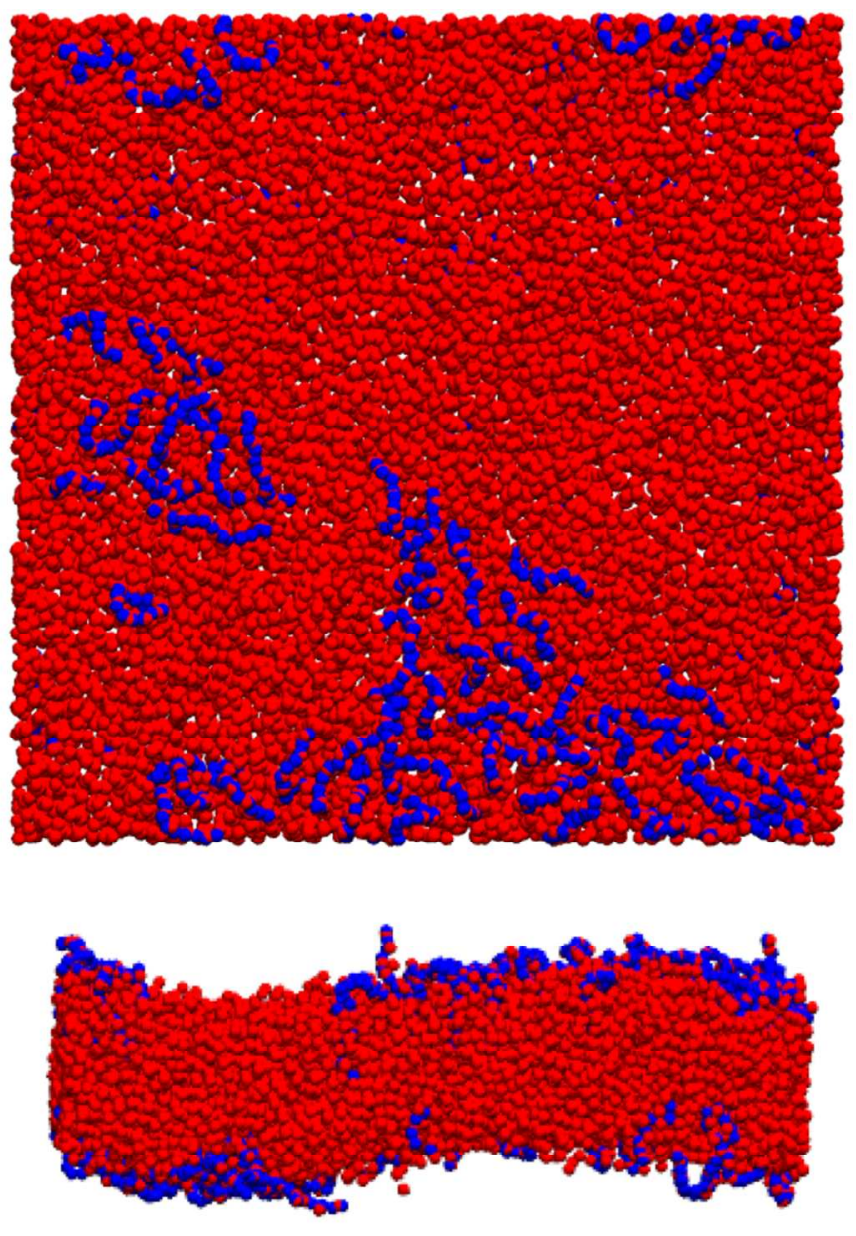

Figure S5: Equilibrium configuration of mixed TX-100/DPPC bilayer. In red are reported the DPPC molecules, while in blue are depicted the TX-100 molecules. 
In the Table S2 the numerical values of Lateral Diffusion Coefficient for the DPPC and TX-100 molecules are reported.

Table S2: Lateral Diffusion Coefficient calculate for the CG bilayer systems.

\begin{tabular}{cccc}
\hline $\begin{array}{c}\mathbf{T} \\
(\mathrm{K})\end{array}$ & $\begin{array}{c}\text { Composition } \\
\mathbf{X}_{\mathrm{TX}-100 / \mathrm{DPPC}}\end{array}$ & $\begin{array}{c}\mathbf{D}(\mathbf{D P P C}) \\
\left(10^{-5} \mathrm{~cm}^{2} / \mathrm{s}\right)\end{array}$ & $\begin{array}{c}\mathbf{D}(\mathbf{T X}-100) \\
\left(10^{-5} \mathrm{~cm}^{2} / \mathrm{s}\right)\end{array}$ \\
\hline 295 & & & \\
& 0.0 & 0.0445 & - \\
& 0.29 & 0.0381 & 0.0321 \\
& 0.56 & 0.0331 & 0.0151 \\
& 0.85 & 0.0284 & 0.0090 \\
& 1.22 & 0.0244 & 0.0125 \\
& & $0.0033^{*}$ & $0.0023^{*}$ \\
\hline
\end{tabular}

* the value is referred to the all atom simulation.

In Tables S3-S5 the numerical values of the order parameter $<\mathrm{P} 2>$ are reported.

Table S3. Order parameter $<\mathrm{P}_{2}>$ of consecutive bonds at the temperature of $295 \mathrm{~K}$.

\begin{tabular}{ccc}
\hline Bond & $\begin{array}{c}<\mathbf{P}_{2}> \\
\chi=0\end{array}$ & $\begin{array}{c}<\mathbf{P}_{2}> \\
\chi=0.122\end{array}$ \\
\hline NC3-PO4 & $-0.147+-0.007$ & $-0.026+-0.015$ \\
PO4-GLY & $0.659+-0.007$ & $0.665+-0.011$ \\
GLY-GLY & $-0.236+-0.007$ & $-0.167+-0.008$ \\
GLY-C1 & $0.554+-0.021$ & $0.499+-0.019$ \\
C1-C2 & $0.572+-0.016$ & $0.488+-0.020$ \\
C2-C3 & $0.495+-0.016$ & $0.392+-0.020$ \\
C3-C4 & $0.344+-0.020$ & $0.250+-0.019$ \\
\hline
\end{tabular}

Table S4. Order parameter $<\mathrm{P}_{2}>$ of consecutive bonds at the temperature of $298 \mathrm{~K}$.

\begin{tabular}{ccc}
\hline Bond & $\begin{array}{c}<\mathbf{P}_{2}> \\
\chi=0\end{array}$ & $\begin{array}{c}<\mathbf{P}_{2}> \\
\chi=0.122\end{array}$ \\
\hline NC3-PO4 & $-0.145+-0.009$ & $-0.022+-0.014$ \\
PO4-GLY & $0.655+-0.007$ & $0.662+-0.012$ \\
GLY-GLY & $-0.235+-0.009$ & $-0.168+-0.009$ \\
GLY-C1 & $0.547+-0.018$ & $0.495+-0.021$ \\
C1-C2 & $0.558+-0.015$ & $0.478+-0.018$ \\
C2-C3 & $0.483+-0.015$ & $0.382+-0.018$ \\
C3-C4 & $0.330+-0.018$ & $0.243+-0.016$ \\
\hline
\end{tabular}


Table S5. Order parameter $<\mathrm{P}_{2}>$ of consecutive bonds at the temperature of $323 \mathrm{~K}$.

\begin{tabular}{ccc}
\hline Bond & $\begin{array}{c}<\mathbf{P}_{2}> \\
\chi=0\end{array}$ & $\begin{array}{c}<\mathbf{P}_{2}> \\
\chi=0.122\end{array}$ \\
\hline NC3-PO4 & $-0.132+-0.007$ & $-0.036+-0.009$ \\
PO4-GLY & $0.633+-0.010$ & $0.634+-0.010$ \\
GLY-GLY & $-0.219+-0.008$ & $-0.164+-0.009$ \\
GLY-C1 & $0.508+-0.027$ & $0.466+-0.027$ \\
C1-C2 & $0.492+-0.017$ & $0.435+-0.022$ \\
C2-C3 & $0.399+-0.014$ & $0.333+-0.018$ \\
C3-C4 & $0.257+-0.019$ & $0.205+-0.015$ \\
\hline
\end{tabular}


In Figure S6 the radial distribution functions, calculated for the polar and aliphatic groups, are reported for the pure bilayer and those one in presence of TX-100, for different temperatures.
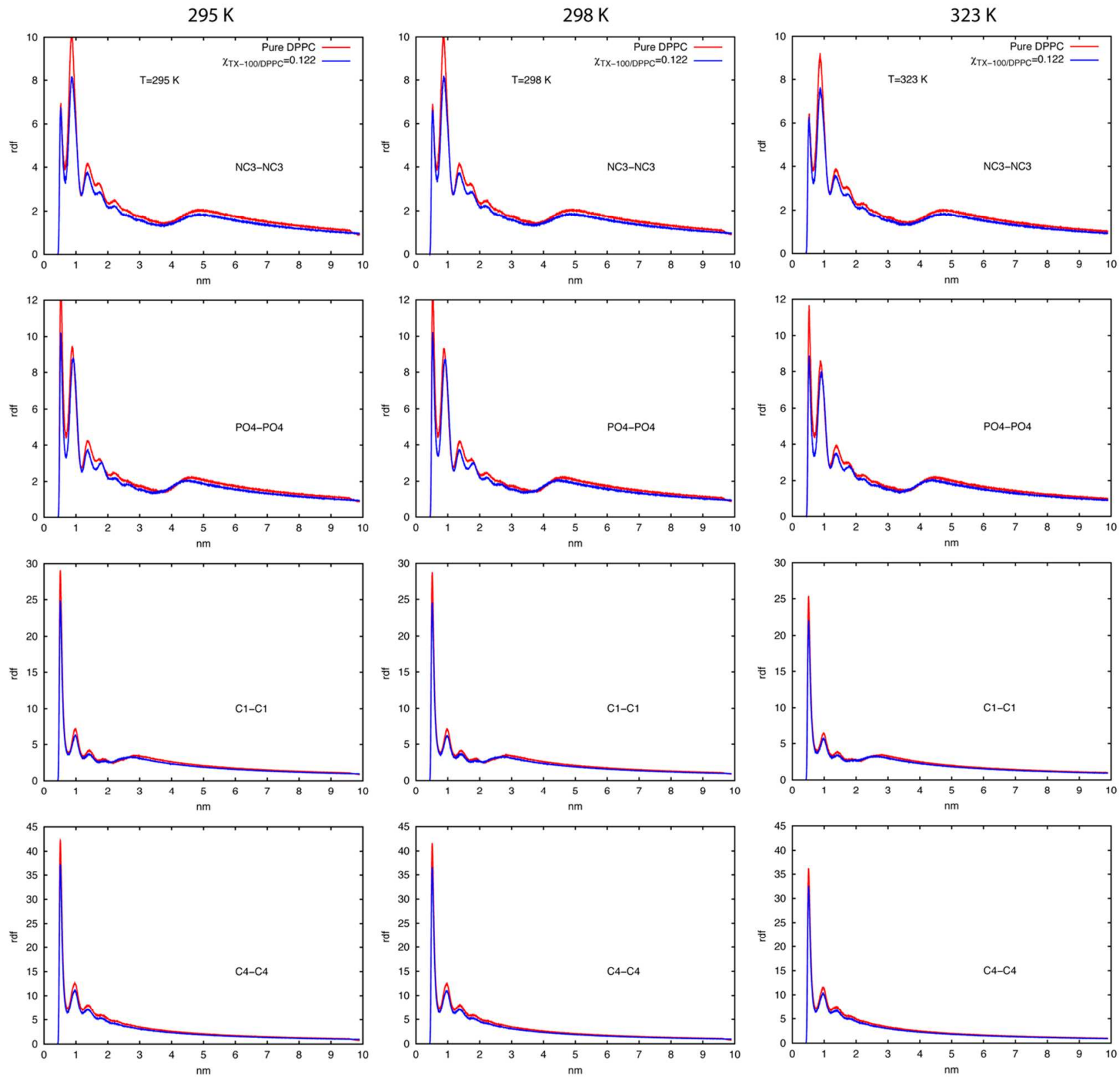

Figure S6: Radial distribution functions for pure DPPC bilayer (red line) and in presence of TX-100 [ $\chi=0.122]$ (blue line), at 295,298 and $323 \mathrm{~K}$. 


\section{References:}

1. Duncan, S. L.; Larson, R. G., Comparing Experimental and Simulated Pressure-Area Isotherms for DPPC. Biochim. Biophys. Acta, Biomembr. 2011, 94 (8), 2965-2986.

2. Rana, F. R.; Mautone, A. J.; Dluhy, R. A., Surface chemistry of binary mixtures of phospholipids in monolayers. Infrared studies of surface composition at varying surface pressures in a pulmonary surfactant model system. Biochemistry 1993, 32 (12), 3169-3177.

3. Nyholm, T.; Slotte, J. P., Comparison of Triton X-100 Penetration into Phosphatidylcholine and Sphingomyelin Mono- and Bilayers. Langmuir 2001, 17 (16), 4724-4730.

4. Dynarowicz-Łatka, P.; Rosilio, V.; Boullanger, P.; Fontaine, P.; Goldmann, M.; Baszkin, A., Influence of a Neoglycolipid and Its PEO-Lipid Moiety on the Organization of Phospholipid Monolayers. Langmuir 2005, 21 (25), 11941-11948.

5. Hunt, R. D.; Mitchell, M. L.; Dluhy, R. A., The interfacial structure of phospholipid monolayer films: an infrared reflectance study. J. Mol. Struct. 1989, 214, 93-109.

6. Taneva, S.; McEachren, T.; Stewart, J.; Keough, K. M. W., Pulmonary Surfactant Protein SP-A with Phospholipids in Spread Monolayers at the Air-Water Interface. Biochemistry 1995, 34 (32), 10279-10289.

7. Tabak, S. A.; Notter, R. H.; Ultman, J. S.; Dinh, S. M., Relaxation effects in the surface pressure behavior of dipalmitoyl lecithin. J. Colloid Interface Sci. 1977, 60 (1), 117-125.

8. Albrecht, O., Experimental study of the stability and metastability of palmitic acid. Thin Solid Films 1989, 178 (1), 93-101.

9. Crane, J. M.; Putz, G.; Hall, S. B., Persistence of Phase Coexistence in Disaturated Phosphatidylcholine Monolayers at High Surface Pressures. Biophys. J. 1999, 77 (6), 3134-3143.

10. Kanintronkul, Y.; Srikhirin, T.; Angsuthanasombat, C.; Kerdcharoen, T., Insertion behavior of the Bacillus thuringiensis Cry4Ba insecticidal protein into lipid monolayers. Arch. Biochem. Biophys. 2005, 442 (2), $180-186$.

11. Shen, Y.; Tang, Y.; Xie, A.; Zhu, J.; Li, S.; Zhang, Y., Studies on behaviors of dipalmitoylposphatidylcholine and bilirubin in mixed monolayer at the air/water interface. Appl. Surf. Sci. 2006, 252 (16), 5861-5867.

12. Lee, Y.-L.; Lin, J.-Y.; Chang, C.-H., Thermodynamic characteristics and Langmuir-Blodgett deposition behavior of mixed DPPA/DPPC monolayers at air/liquid interfaces. J. Colloid Interface Sci. 2006, 296 (2), $647-654$.

13. Skibinsky, A.; Venable, R. M.; Pastor, R. W., A Molecular Dynamics Study of the Response of Lipid Bilayers and Monolayers to Trehalose. Biophys. J. 2005, 89 (6), 4111-4121.

14. Adhangale, P. S.; Gaver, D. P., Equation of state for a coarse-grained DPPC monolayer at the air/water interface. Mol. Phys. 2006, 104 (19), 3011-3019.

15. Marrink, S. J.; de Vries, A. H.; Mark, A. E., Coarse Grained Model for Semiquantitative Lipid Simulations. J. Phys. Chem. B 2004, 108 (2), 750-760. 